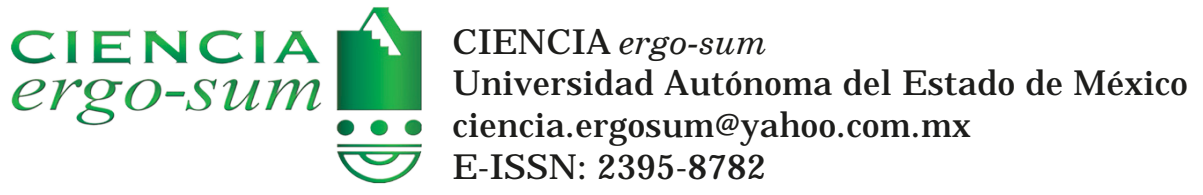

\title{
Rayos X y resonancia magnética en la entomología: principios y aplicaciones
}

\author{
Maurice-Lira, J orge Víctor; Valdez-Carrasco, J orge Manuel \\ Rayos $X$ y resonancia magnética en la entomología: principios y aplicaciones \\ CIENCIA ergo-sum, vol. 29, núm. 1, marzo-junio 2022 | e152 \\ Ciencias Naturales y Agropecuarias \\ Universidad Autónoma del Estado de México, México
}

Esta obra está bajo una Licencia Creative Commons Atribución-NoComercial-SinDerivar 4.0 Internacional.

$$
\text { (c) (\$) }(-
$$

Maurice-Lira, J . V. y Valdez-Carrasco, J . M. (2022). Rayos X y resonancia magnética en la entomología: principios y aplicaciones. CIE N CI A ergo-sum, 29(1). https://doi.org/10.30878/ces.v29nla8 


\title{
Rayos $\mathrm{X}$ y resonancia magnética en la entomología: principios y aplicaciones
}

\section{$\mathrm{X}$-rays and magnetic resonance in the entomology: Principles and applications}

\author{
Jorge Victor Maurice-Lira \\ Colegio de Postgraduados, Campus Montecillo, México \\ jvml333@gmail.com \\ (D) http://orcid.org/0000-0001-7166-5569 \\ Jorge Manuel Valdez-Carrasco \\ Colegio de Postgraduados, Campus Montecillo, México \\ jvaldez@colpos.mx \\ (D) http://orcid.org/0000-0003-4804-4435
}

Recepción: 17 de julio de 2020

Aprobación: 12 de enero de 2021

\section{RESUMEN}

Se describe brevemente la historia y principios de la generación de imágenes mediante rayos $\mathrm{X}$ y resonancia magnética por medio de una revisión bibliográfica. También, se analizan sus aplicaciones en la entomología y otros campos agronómicos, además de las perspectivas para investigaciones futuras. Se concluye que las metodologías analizadas no son destructivas puesto que permiten dar seguimiento al mismo individuo con imágenes y modelos computarizados en dos y tres dimensiones para el estudio de células, tejidos y órganos en insectos vivos. Estas técnicas contribuyen a la comprensión morfológica, fisiológica y ecológica de los insectos en diferentes ambientes o tratamientos.

Palabras ClaVe: imagenología aplicada a la entomología, morfología de insectos, rayos X, RMN.

\begin{abstract}
This literature review briefly describes the history and principles of X-ray and magnetic resonance imaging, its applications in entomology and other agronomic fields are analyzed, besides the perspectives for future research. It is concluded that the analyzed methodologies are non-destructive, they allow to give follow-up to the same individual through images and computerized models in two and three dimensions for the study of cells, tissues and organs in living insects. These techniques contribute to the morphological, physiological and ecological understanding of insects in different environments or treatments.
\end{abstract}

KEYWORDS: Imaging applied to entomology, insect morphology, X-rays, NMR.

\section{INTRODUCCIÓN}

Los análisis histológicos son muy utilizados en el estudio de estructuras internas y órganos de insectos, pero mediante estas técnicas se obtienen imágenes con distribuciones poco representativas de las que se observarían en uno vivo. En este sentido, sacrificar al insecto imposibilita observar los cambios que ocurren durante su desarrollo o después de que se le haya aplicado algún tratamiento (Aguayo et al., 1986; Schilling et al., 2012).

Las metodologías empleadas para la generación de imágenes en el campo médico, como los rayos X, han permitido estudiar con mayor profundidad la biología de los insectos desde enfoques anatómicos, morfológicos, etológicos y fisiológicos en diferentes ambientes, así como durante su desarrollo ontogénico y fenológico (Salinas et al., 2016; Keszthelyi et al., 2020a).

El avance tecnológico y las variantes metodológicas basadas en los rayos $\mathrm{X}$ han perfeccionado la generación de información a partir de imágenes con gran contraste y calidad (Lee y Socha, 2009). En la actualidad, se pueden obtener tomografías con una resolución micrométrica y también de modelos computarizados en 3D (Keszthelyi et al.,2020a). 
Por medio de estas herramientas se puede visualizar y estudiar la morfología de los insectos, así como describirlos con más detalle para lograr una identificación precisa en trabajos de taxonomía y sistemática (Triplehorn y Johnson, 2005; Ijiri et al., 2018); asimismo, se pueden investigar sus comportamientos y desarrollo fisiológico con mayor exactitud.

Por otra parte, la aplicación de metodologías basadas en los rayos X puede expandirse hacia otros campos agronómicos como la evaluación del impacto de las plagas en los frutos, semillas u otras estructuras vegetales de importancia económica (Aristizábal-Torres, 2007). Lo anterior es con el objetivo de monitorear la presencia de las plagas en los cultivos o de evaluar la calidad de los frutos y semillas en el manejo de poscosecha.

Otro método de imagen que ha cobrado gran relevancia es el uso de la resonancia magnética nuclear (RMN). Del mismo modo que los rayos X, esta tecnología no es invasiva y se puede realizar in vivo, por lo que permite repetir los estudios varias veces en el mismo espécimen. La RMN brinda ventajas en el seguimiento del desarrollo de los insectos después de haberles aplicado tratamientos que se deseen evaluar. También se puede obtener información adicional: cambios en la densidad o composición de tejidos o modificaciones químicas en estructuras específicas del insecto, incluso en nivel celular (Nelson y Cox, 2009; Keszthelyi et al., 2020a).

Este artículo describe brevemente la historia y los fundamentos de la generación de imágenes mediante rayos X y RMN, dos metodologías utilizadas en el campo médico y se analizaron sus aplicaciones en la entomología. Asimismo, se describen los principales usos, algunas de sus variantes más modernas y algunas perspectivas de investigaciones futuras en la entomología y otros campos agronómicos.

\section{RAYOS X}

\section{1. Breve historia}

En noviembre de 1895, el físico alemán Wilhelm Roentgen estudiaba la producción de rayos catódicos a través de grandes tubos Crookes fluorescentes. Mientras preparaba su experimento, casi por accidente, descubrió que al encender los tubos Crookes una placa de platinocianuro de bario fluorescente cercana comenzó a brillar. Tras observar el fenómeno tomó la placa y la acercó a los tubos Crookes encendidos: se percató que la intensidad del brillo de la placa aumentaba conforme la acercaba más a los tubos. Había descubierto la procedencia de esa luz desconocida, a la que nombró "luz X" (Salinas et al., 2016).

Este fenómeno ya había sido notado por otros científicos, pero fue Roentgen quien lo estudió a profundidad y antes de terminar ese año ya había descrito todas las propiedades conocidas sobre los rayos X. Gracias a eso ganó el Premio Nobel de Física en 1901 (Salinas et al., 2016).

En 1903, la Sociedad de Silvicultura Sueca se interesó por el uso de los rayos X en evaluaciones de semillas forestales, pero en ese momento no se le brindó mayor relevancia. Ahora bien, los entomólogos alemanes sí identificaron con rapidez la aplicación de los rayos X como un método para detectar insectos en el interior de las semillas y diferentes órganos vegetales. En 1970 fue cuando la Asociación Oficial de Analistas de Semillas de Estados Unidos publicó el manual Análisis radiográfico de semillas de cultivos agrícolas y forestales donde se describía el uso de los rayos X para la evaluación de semillas en el sector agrícola y forestal. Esto es, fueron los entomólogos quienes desde el principio adoptaron las tomografías por rayos X para el estudio en la morfología y ecología de los insectos (Salinas et al., 2016).

\section{2. Principio}

Los rayos X corresponden a la radiación electromagnética con longitudes de onda de 1 a $100 \mathrm{~nm}$, cuya energía de sus fotones es muy superior al espectro de luz visible que oscila entre las longitudes de onda de 380 y $750 \mathrm{~nm}$ 
(Nelson y Cox, 2009). Esta energía se produce cuando se calienta el filamento de un cátodo para activar sus electrones a un alto nivel de energía y después los electrones son disparados a través de un tubo de rayos $\mathrm{X}$ vacío en dirección hacia un ánodo. Cuando los electrones colisionan contra el ánodo en un "punto focal" se producen los rayos $\mathrm{X}$, los cuales son redirigidos a través de una ventana hecha de berilio. En ese momento es cuando se produce un haz cónico dirigido hacia la muestra (Salinas et al., 2016).

La obtención de radiografías o tomografías por rayos X se fundamenta en la emisión de un haz de radiación electromagnética a través de un cuerpo (región anatómica o una muestra). En el caso de las radiografías clásicas, la radiación que atraviesa el cuerpo estudiado revela la imagen en una película radiográfica (Magnotta et al., 2014), mientras que en las tomografías computarizadas la radiación electromagnética que atraviesa la muestra analizada es detectada por un sensor conectado a un ordenador encargado de procesarla para digitalizar la imagen (Magnotta et al., 2014).

En ambos casos se crean imágenes en escalas de grises, basadas en la cantidad de rayos X que atraviesan tejidos con diferente densidad: a eso se le denomina densidad del agua, porque este elemento se coloca como punto de referencia. La intensidad del gris depende del haz de rayos X que alcance o exponga a la película radiográfica o al sensor, el cual es mayor cuando atraviesa tejidos poco densos y menor cuando el tejido es muy denso (Magnotta et al., 2014; Rühr y Lambertz, 2019).

Los tejidos más densos como el esqueleto (endo o exoesqueleto) absorben gran cantidad de radiación electromagnética; por otra parte, el tejido adiposo es poco denso y absorbe muy poca radiación. Por lo tanto, cuando la cantidad de rayos $\mathrm{X}$ absorbidos es mayor, se produce una imagen más blanca; en contraste, cuando los rayos $\mathrm{X}$ atraviesen sin ser absorbidos por el tejido, la imagen obtenida es más oscura (Magnotta et al., 2014). En relación con esto, el color más negro corresponde al aire (el cual no absorbe nada o casi nada de la radicación), el tejido adiposo genera imágenes poco más claras que el aire debido a que es muy poco denso, el tejido muscular varía con base en su densidad y las estructuras más densas como hueso, depósitos de calcio o metales absorben casi toda o toda la radiación (Magnotta et al., 2014).

Tecnologías más modernas que se basan en este principio han aumentado la precisión y el contraste de las imágenes. En un principio se utilizaban placas intensificadoras de fósforo que emitían luz al ser alcanzadas por los rayos X no absorbidos; de este modo, la luz intensificada aumentaba el contraste en las películas radiográficas o en las imágenes digitales.

En la tomografía computarizada (TC) se utiliza una cabina para que la radiación electromagnética sea mejor dirigida hacia los puntos de mayor interés en el estudio imagenológico, las TC dinámicas o de multicorte permiten tomar imágenes en movimiento, además de múltiples imágenes desde diferentes ángulos para reconstruirlas por computadora mediante algoritmos matemáticos que permitan generar modelos en $3 \mathrm{D}$ de órganos, estructuras o cuerpos de interés (Magnotta et al., 2014; Wakonig et al., 2019). Esto último ha traído grandes beneficios debido a que algunos sistemas convencionales de obtención de imágenes con rayos $\mathrm{X}$ ajustan la dirección del flujo electromagnético mediante espejos para concentrar el haz en la muestra lo mejor posible (Andrews et al., 2011), pero el uso de espejos puede causar distorsiones en la imagen respecto a la muestra (Wakonig et al., 2019). La psicografía de rayos X permite analizar mediante algoritmos matemáticos los patrones de interferencia de una muestra iluminada por varios haces de rayos X para poder detectar la posición exacta de cada patrón de difracción de la muestra. Esta técnica aumenta la precisión de las imágenes sin distorsionarlas aun a escalas micrométricas (Wakonig et al., 2019).

Otro reto en el análisis de imágenes generadas por radiación electromagnética de rayos X es la visualización de estructuras con buen contraste a partir de integumento transparente (como las alas de insectos); con densidad es apenas superior a la del aire, por lo que generan imágenes poco contrastadas y casi imperceptibles (Rühr y Lambertz, 2019). En estos casos se pueden cubrir las muestras con una fina capa de átomos de oro para brindar mayor densidad a las estructuras y lograr imágenes mucho más detalladas y así lograr tomografías microcomputarizadas de muy alta definición (Rühr y Lambertz, 2019). 
El recubrimiento en oro es ampliamente utilizado para la imagenología por microscopía electrónica de barrido (SEM por sus siglas en inglés) y se ha demostrado que aumenta el nivel de contraste en la reconstrucción de modelos en 3D con tomografía microcomputarizada de rayos $\mathrm{X}$, ya que se pueden obtener imágenes tanto superficiales como del tejido y de estructuras internas (Rühr y Lambertz, 2019). Esto es de suma importancia en la taxonomía debido a que la venación de las alas tiene un uso habitual para lograr la clasificación de insectos en nivel de especie.

Otro mecanismo que permite crear modelos en 3D de muy alta calidad es la combinación de la tomografía computarizada de rayos X con la fotografía digital, que utiliza como base las medidas de la muestra para obtener imágenes volumétricas y se obtienen también detalles de la textura y color de las superficies (Ijiri et al., 2018). Esta metodología utiliza las bases de la microtomografía computarizada $(\mu \mathrm{CT})$ que se suma a la generación de múltiples fotografías de alta resolución tomadas con cámaras digitales desde diferentes ángulos de rotación analizados mediante vectores por un ordenador.

\section{3. Aplicación en estudios anatómicos}

Cualquier estudio entomológico requiere identificación taxonómica o morfológica de estructuras específicas del cuerpo de los insectos. Metodologías basadas en rayos X permiten obtener imágenes precisas del cuerpo completo de un insecto o de estructuras de interés como musculatura, tejido graso y órganos.

Algunas variantes metodológicas de los rayos $\mathrm{X}$ son tan sensibles que se pueden identificar compuestos moleculares o celulares; por ejemplo, mediante la técnica de difracción de rayos $\mathrm{X}$ por radiación sincrónica se pueden estudiar compuestos moleculares (como proteínas) de los músculos u otro tipo de tejidos en los insectos (Al-Khayat et al., 2003; Iwamoto, 2018; Knupp et al., 2019). Este tipo de estudios morfológicos permite dar seguimiento a la conformación molecular de los tejidos de insectos vivos (Iwamoto, 2018) y brinda información inaccesible mediante metodologías clásicas. En este sentido, la morfología de las estructuras internas de la genitalia de algunos insectos (como los del orden Coleóptera) varían dependiendo de la especie y son esenciales para su identificación taxonómica.

Extraer la genitalia puede significar una tarea compleja en insectos de tamaño milimétrico, pero con difracción de rayos $\mathrm{X}$ por radiación sincrónica se podría visualizar, ya que son estructuras recubiertas de tejido graso y muscular. No obstante, la alta energía de los microhaces de rayos X sí puede alterar las biomoléculas de especímenes hidratados, por lo que es recomendable cambiar la posición del espécimen para que el haz de rayos $\mathrm{X}$ no se mantenga sobre una misma región de su cuerpo.

Otra metodología que permite estudiar la anatomía y morfología del cuerpo completo o secciones de interés de los insectos es la reconstrucción digital de modelos en 3D, la cual facilita el estudio en diversas áreas de la entomología como en sistemática, taxonomía, estudios del desarrollo ontogénico, análisis funcionales y procesos evolutivos (Triplehorn y Johnson, 2005; Betz et al., 2007; Büsse et al., 2015; Büsse et al., 2016; Ijiri et al., 2018; Bukejs et al., 2020).

La $\mu \mathrm{CT}$ de alta resolución permite reconstruir modelos en 3D de estructuras externas e internas (Büsse et al., 2016). Este tipo de imagenología es de gran apoyo para esquematizar y mejorar la comprensión del desarrollo y anatomía de los insectos (Bukejs et al., 2020). Büsse et al. (2015) estudiaron la musculatura torácica de dos especies de Epiophlebia. Sus análisis demostraron que este género poseía una morfología torácica ancestral con 75 músculos, el mayor número encontrado en el orden Odonata. Este trabajo demostró que mediante la reconstrucción de las estructuras internas de una especie en concreto se puede dilucidar sus procesos evolutivos.

La microscopía por rayos $\mathrm{X}$ de transmisión es una técnica con un grado de precisión nanométrico capaz de brindar imágenes de estructuras celulares que puede combinarse con reconstrucción de modelos en 3D (Andrews et al., 2011). Incluso, es posible identificar algunas biomoléculas al contrastar la diferencia de absorción 
de la radiación electromagnética de cada átomo (Andrews et al., 2011). Esta técnica, así como el análisis de la difracción de los rayos X, permite identificar y cuantificar la estructura de las biomoléculas de diversos tejidos (Al-Khayat et al., 2003).

Conocer la composición de los integumentos permite inferir la función de diversas estructuras anatómicas (Büsse y Gorb, 2018; Rühr y Lambertz, 2019). En su caso, la larva de Erythromma najas (Odonata: Coenagrionidae) muestra una transición membranosa de la proteína resilina entre el anteclipeo y el labrum, mientras que los incisivos mandibulares son áreas muy rígidas altamente esclerosadas. Estas estructuras se adaptan con base en sus funciones: mientras que la resilina actúa como almohadilla de protección contra daños por impacto, los incisivos requieren una mayor rigidez para soportar la presión ejercida al triturar a las presas. La combinación de varias metodologías como la tomografía de rayos $\mathrm{X}$ de alta resolución permite identificar los compuestos químicos o moleculares de las estructuras en nivel tisular, celular y molecular (Al-Khayat et al., 2003; Büsse y Gorb, 2018).

\subsection{Aplicación en estudios fisiológicos}

En 2009 se diseñó una estrategia directa para observar por primera vez el flujo de la hemolinfa en el interior del cuerpo de Schistocerca americana. En esta técnica in vivo descrita por Lee y Socha (2009) utilizaron microburbujas de alto contraste con diámetros de 10 a $15 \mu \mathrm{m}$, las cuales se observaron con éxito gracias a la imagenología de sincrotón de rayos $\mathrm{X}$ de contraste de fases. Esta técnica solucionó el gran reto que significaba visualizar la hemolinfa y su flujo en insectos debido a la escala espacio temporal mínima de $50 \mu \mathrm{m}$ y $0.1 \mathrm{~s}$ requerida para comprender su fisiología. En este trabajo lograron visualizar claramente algunas estructuras internas como los sacos aéreos, segmentos de la tráquea y el borde ventral del diafragma, donde se aglomeraron las burbujas, incluso se observaron compartimentos de la región dorsal del corazón. También se descubrió que, gracias a la dilatación y compresión del diafragma, la hemolinfa fluye hacia la cabeza a través de los conductos a una velocidad de $9.5 \mathrm{~mm} / \mathrm{s}$.

\subsection{Aplicación en estudios del comportamiento}

La imagenología generada por rayos X puede extenderse al estudio del comportamiento de los insectos en sus ambientes naturales. La imagenología cinética basada en el movimiento permite identificar la presencia de plagas en tejido vegetal de manera no destructiva para estudiar su dinámica en el interior de las plantas hospederas (Keszthelyi et al., 2020a). Las larvas de algunas especies pueden habitar largos periodos y sólo tener movimientos moderados de apenas pocos milímetros, ya que los hospederos representan un refugio contra las condiciones climáticas adversas. Durante ese tiempo, las larvas permanecen en diapausa y su metabolismo disminuye, por lo que tienen muy poca actividad dentro de la planta, mientras que el mayor movimiento podría darse en periodos con mayor biomasa vegetal. En esta metodología se aplica el medio de contraste intravenoso para visualizar los canales realizados por las larvas (Keszthelyi et al., 2020b).

Pero el uso de esta técnica no se limita a la observación de los órganos vegetales parasitados por insectos, ya que su uso puede enfocarse también en la evaluación de la maduración y calidad de frutos, semillas y otros órganos vegetales de interés (Márquez-Cardozo et al., 2009; Salinas et al., 2016). Además, gracias a que las evaluaciones no son destructivas, se puede monitorear la presencia de insectos que causan daños en el interior de los órganos vegetales.

El análisis de frutos que desarrolló Márquez-Cardozo et al. (2009) para evaluar daños y presencia de plagas requirió una distancia entre la fuente de rayos X y la muestra de $650 \mathrm{~mm}$. Estos autores también utilizaron dos pantallas luminiscentes de oxisulfuro de gadolinio para intensificar el contraste de las imágenes. Ambas películas se colocaron a cada lado de la película celuloide recubierta con bromuro de plata. 


\section{Resonancia magnética nUClear}

\section{1. Breve historia}

En 1986 Aguayo et al., utilizaron por primera vez la resonancia magnética nuclear (RMN) acoplada a un microscopio para observar los óvulos de la rana de uñas africana (Xenopus laevis) y sus orgánulos diferenciados. Este trabajo demostró la viabilidad del método para obtener imágenes de estructuras muy pequeñas de modo no invasivo; asimismo, abrió camino para usar la RMN en nuevos campos de la biología, más allá de la medicina, debido a que no sólo sirve para observar estructuras de gran tamaño como órganos humanos o de animales superiores, sino que demostró su factibilidad para diferenciar estructuras en el interior de células, con base en las diferencias de la composición química entre los orgánulos. Por ejemplo, el agua en el interior del núcleo tiene diferente concentración de protones $\mathrm{H}+$ que el agua contenida en el citoplasma (Aguayo et al., 1986), por lo que la variación de protones, sumada a su tiempo de relajación tras ser estimulados electromagnéticamente, permite diferenciar las estructuras intracelulares, lo que también aplica para tejidos adiposos (Schilling et al., 2012). Por otra parte, este método no invasivo permite estudiar organismos sin la necesidad de matarlos y disectarlos (Aguayo et al., 1986).

Poco tiempo después se comenzó a utilizar este método para el estudio no destructivo ni invasivo en la entomología (Seo, 2018), que demostró tener grandes ventajas para documentar imágenes precisas de la morfología interna de los insectos respecto a las técnicas clásicas que requerían abundantes especímenes para su disección y análisis (Schilling et al., 2012). Con la RMN se hizo posible obtener información y documentar al mismo insecto varias veces para darle seguimiento en diferentes edades durante su desarrollo fisiológico, así como durante etapas fenológicas específicas como el desarrollo de los gametos o durante procesos específicos como la acumulación y flujo de las reservas de energía en función de actividades cotidianas como el vuelo (Favila et al., 2004).

Por otra parte, la RMN permite documentar la información tanto cualitativa (mediante imágenes) como cuantitativamente (el contenido molecular de los tejidos), por ejemplo, del contenido lipídico de tejidos adiposos que se ocupan como reservorios de energía durante el desarrollo larvario u otros mecanismos de supervivencia durante periodos de hibernación en estaciones invernales (Schilling et al., 2012).

\section{2. Principio}

La RMN permite analizar estructuras orgánicas de forma no invasiva en un organismo mediante una propiedad cuántica denominada espín nuclear, que algunos núcleos atómicos tienen al ser sometidos a un campo magnético. Esta propiedad consiste en la orientación en la que giran los protones de estos elementos, lo que a su vez les genera un dipolo magnético, pero en ausencia de un campo magnético no tienen una dirección definida, puesto que se encuentran en un estado sin energía diferenciada (Nelson y Cox, 2009).

Cuando una muestra es sometida a un campo electromagnético constante, a una frecuencia de resonancia adecuada en pulsos breves, los dipolos se orientan en dirección paralela (espín $\alpha$ ) o antiparalela (espín $\beta$ ) al campo energético aplicado durante la imagenología. La energía aplicada será absorbida y acumulada por los núcleos orientados hasta el punto de que los espines $\alpha$ cambian su dirección (hacia $\beta$ ). Ese punto es denominado resonancia, mientras que la frecuencia de resonancia es aquella energía con la que los protones con espín $\alpha$ cambian a espín $\beta$. Más adelante, se mide el "tiempo de relajación", que es el necesario para que los átomos regresan a su posición original que tenían antes de la aplicación del campo electromagnético (Nelson y Cox, 2009).

Con base en la relación de la concentración de protones y su tiempo de relajación, se puede cuantificar de manera directa el flujo energético para establecer un modelo de proyección de acuerdo con el procesamiento basado en las fuentes de corriente. Dicho modelo permite calcular la información de la estructura, posición, volumen y pH (con base en concentración de átomos de $\mathrm{H}+$ ) y después se procesa la información para generar 
un campo vectorial que puede trazarse en una tomografía en 2D o bien en un modelo en 3D generado por computadora (Rodríguez-Camacho et al., 2011). Sin embargo, para obtener un modelo en 3D es necesario producir imágenes de segmentos, incluso de compartimentos internos de la muestra (Silva-Pereyra, 2011), y para asegurar una descripción más detallada de las estructuras analizadas se debe someter la estimación de las fuentes y flujos de energía a análisis estadísticos complejos que no se discutirán en este artículo, pero la mayoría de softwares empleados cuentan con las herramientas necesarias para realizarlas con propósitos de visualización de imágenes detalladas (Silva-Pereyra, 2011).

Gracias a estos estudios es posible dar seguimiento a los cambios en la morfología de todos los órganos de un insecto, por lo que es factible monitorear las dinámicas de consumo y uso de la energía de reserva en las diferentes etapas fisiológicas de los insectos (Schilling et al., 2012). Por igual, se puede relacionar la estructura y disposición de reservas de tejido graso en función de su comportamiento durante tiempos prolongados o en diferentes condiciones climáticas.

Estos beneficios no los podían brindar las técnicas de la entomología clásica, ya que requerían sacrificar múltiples especímenes en diferentes etapas de su desarrollo fisiológico y las mediciones que se realizaban eran indirectas, basadas en correlaciones de tamaño o biomasa de referencia de varios individuos con características similares (misma especie, mismo origen, etc.). Es decir, eran meras aproximaciones sujetas a variables extrínsecas incontrolables que pudieran afectar el desarrollo de alguno de los individuos de referencia, lo que disminuye la exactitud de los datos estimados. No obstante, la RMN brinda la posibilidad de dar seguimiento al estudio de los órganos, estructuras y tejidos de los insectos en diferentes etapas de su desarrollo sin la necesidad de matarlos con una resolución de hasta $31 \mu \mathrm{m}$ (Schilling et al., 2012).

La resonancia magnética de alta resolución permite observar estructuras incluso en un nivel molecular. Al respecto, la variante resonancia magnética funcional permite generar imágenes en secuencia con un corto intervalo entre cada imagen, aunque la resolución de estas imágenes se reduce a $1 \mathrm{~mm}^{3}$, aproximadamente (Rodríguez-Camacho et al., 2011), pero se puede editar la secuencia de imágenes para observar el movimiento de las estructuras estudiadas.

\subsection{Aplicaciones}

La RMN ha sido requerida a menudo por los entomólogos en diversas áreas como morfología general, parasitología, estudio del desarrollo, metabolismo e incluso biomagnetismo (Hart et al., 2003), y, debido a que se puede estudiar al mismo espécimen varias veces sin necesidad de sacrificarlo, es posible observar su evolución durante periodos específicos (Aguayo et al., 1986; Schilling et al., 2012).

Favila et al., (2004) examinaron los órganos y estructuras internas de machos y hembras adultos de las especies Canthon cyanellus cyanellus. Identificaron las glándulas pigidiales en ambos sexos, los ovarios en las hembras y el edeago en los machos, además analizaron su potencial como indicadores de la fase reproductiva en la que se encuentran los individuos. Sus estudios mostraron, por ejemplo, que los órganos de las hembras varían conforme con su estado de madurez sexual. En el caso de la glándula pigidial de hembras inmaduras se observa turgente y brillosa en la imagen por RMN, mientras que en las hembras maduras turgente, pero no brillosa. Por su parte, en las hembras que recién habían copulado no se observó la glándula pigidial, pero sí un espermatóforo turgente y brilloso. Sus resultados permiten estudiar el funcionamiento de las glándulas pigidiales antes, durante y después de la cópula y durante la formación de las pelotas de cría debido a que la glándula pigidial secreta diversos compuestos cetónicos que inhiben el crecimiento de microorganismos patógenos para las larvas. En todos los casos el tejido graso está distribuido en toda la cavidad abdominal con excepción de las hembras inmaduras y en aquellas que estaban próximas a la oviposición. En machos no observaron una importante diferenciación en las estructuras internas tan sólo algunas estructuras genitales con mayor claridad en adultos maduros. 
Este tipo de trabajos brinda la oportunidad de analizar los cambios de las estructuras y órganos de las especies de insectos en diferentes estados de madurez o etapas sexuales y gracias a que la RMN no es una técnica invasiva se puede dar seguimiento al mismo espécimen durante toda su vida o bien durante periodos específicos.

Otro trabajo que muestra los cambios en la estructura interna de algunos insectos es el de Schilling et al., (2012), quienes evaluaron la composición, distribución y diferenciación del contenido de grasa y agua en diferentes órganos de Ips typographus antes y después del vuelo. Sus resultados demostraron que el mayor reservorio de grasa como fuente de energía se localizó en el abdomen y mediante espectroscopía por RMN se comprobó cómo disminuye la concentración de grasa y agua después del vuelo respecto a la que es previa a éste.

Otra variante de la RMN es la resonancia paramagnética de electrones (RPE), la cual recién fue propuesta para estudios por imagenología de insectos. Charlier et al., (2020) utilizaron los complejos de melanina-quitina presentes en el exoesqueleto de la abeja melífera Apis mellifera para estudiar su morfología y distribución del complejo molecular. Sus resultados demostraron que el complejo de melanina y quitina se distribuye por toda la región periférica del cuerpo, correspondiente a la cutícula del exoesqueleto. Esto permitió analizar toda su morfología, lo cual sugiere que esta metodología es apta para observaciones morfológicas de exoesqueletos en diversos insectos. No obstante, a diferencia de la RMN, en este caso se utilizaron especímenes liofilizados y no insectos vivos.

El estudio por RPE permite utilizar el análisis de los radicales libres para reconstruir imágenes volumétricas en 3D. Los radicales libres, a diferencia de los complejos de melanina-quitina, se encontraron tanto en la periferia como en el centro de la cabeza. Este tipo de estudios permite crear modelos que permitan la observación y análisis de estructuras en diferentes planos y con diferentes cortes tanto de estructuras internas como externas.

Otro campo de aplicación de la RMN es el agroalimentario. Tiene su origen en 1977 cuando se publicó por primera vez la imagen de un limón generada con RMN. A partir de ese momento esta metodología ganó gran relevancia en el sector agroalimentario y manejo de poscosecha. La RMN de baja resolución se acota a escalas macroscópicas como el contenido de aceites y grasas de algunos frutos, semillas, carne o cereales, entre otros alimentos, o contenido de humedad de alimentos deshidratados, mientras que el estudio de RMN de alta resolución permite identificar estructuras moleculares como se ha mencionado en trabajos descritos (Aristizábal-Torres, 2007). Sin embargo, su uso se amplía al estudio de la calidad de los frutos o a la evaluación de los daños en frutos por plaga, patógenos o condiciones climáticas (Aristizábal-Torres, 2007).

El estudio de productos agrícolas y su manejo de poscosecha es de gran interés económico y su evaluación es de suma importancia, por lo que al representar una metodología no destructiva la RMN permite, al igual que con insectos, evaluar su comportamiento y manejo durante periodos prolongados sin causar perjuicios a los frutos. Con base en esto, es posible evaluar el manejo de poscosecha de los frutos sometidos a diferentes condiciones de almacenamiento, aunado a que su uso es una herramienta muy útil en el control de calidad (Aristizábal-Torres, 2007).

\section{AnÁlisis PROSPECTIVo}

El estudio de especímenes de insectos mediante las dos metodologías no destructivas descritas abre paso a estudios más complejos de la fisiología de los insectos sometidos a diferentes tratamientos. Lo anterior con un nuevo enfoque orientado a dar seguimiento continuo a los especímenes con el objetivo de observar los cambios morfológicos, anatómicos, fisiológicos o moleculares de tejidos y órganos específicos.

Cualquier investigación orientada al control de plagas requiere de una identificación taxonómica previa, así como del análisis de la biología y etología (comportamientos o hábitos) de los insectos en ambientes naturales. Las metodologías no invasivas, entonces, permitirían detallar estructuras externas e internas necesarias para la identificación de las especies (Sarnat et al., 2016); también se puede observar el desarrollo fisiológico, así como los comportamientos y hábitos alimenticios o reproductivos de las especies en sus ambientes naturales aun si los especímenes se encuentran dentro del tejido vegetal como semillas, tallos y hojas (Keszthelyi et al., 2020b). 
Equipos portátiles de rayos X pueden ser claves en la evaluación de la presencia de insectos de interés económico y gastronómico como Comadia redtenbacheri y Aegiale hesperiaris localizados en las raíces y la penca de maguey respectivamente. Esta evaluación sería con la finalidad de no dañar la planta hospedera a menos de que se tenga certeza de la presencia de los gusanos comestibles. De igual manera, se pueden aprovechar estas herramientas para el muestreo de la presencia de insectos plaga como minadores en cultivos. Así pues, se podría realizar un manejo integrado y desarrollar tácticas precisas con base en la población de insectos plaga presente en el cultivo.

Otros trabajos enfocados en los efectos que algunos microorganismos entomopatógenos desencadenan en los insectos plaga pueden apoyarse también de los rayos X y la RMN. Algunos hongos entomopatógenos inducen cambios fisiológicos, anatómicos o en el comportamiento de los insectos infectados y aún no es evidente el porqué. Los estudios que no requieren matar al insecto brindan la oportunidad de visualizar el proceso de infección para comprender mejor el mecanismo de infección. Por otra parte, hay interacciones simbióticas mutualistas entre insectos y microorganismos que también inducen cambios fisiológicos en el desarrollo embrionario de los insectos. Estos efectos hoy en día son poco claros y los estudios no invasivos pueden ser la clave para identificar los cambios moleculares y morfológicos resultantes de dichas interacciones (Menezes et al., 2015).

En trabajos enfocados en el desarrollo de robots inspirados en la bioingeniería de los insectos, el diseño de modelos en 3D permitiría visualizar y comprender mejor las estructuras y mecanismos de locomoción o aerodinámica (Zhakypov et al., 2019; Chitsaz et al., 2020).

De acuerdo con lo expuesto, este artículo permite identificar algunas oportunidades de estudio y enfoques que la resonancia magnética nuclear y las tomografías por rayos $\mathrm{X}$ brindan en diversos campos de la entomología aplicada.

\section{Agradecimientos}

Agradecemos la minuciosa labor de los árbitros por aportar mejoras a la estructura del manuscrito, ya que permitieron construir un enfoque más profundo para su análisis, así como también a Conacyt por el financiamiento que ha permitido la elaboración de este trabajo.

\section{REFERENCIAS}

Aguayo, J. B., Blackband, S. J., Schoeninger, J., Mattingly, M. A., \& Hintermann, M. (1986). Nuclear magnetic resonance imaging of a single cell. Letters to Nature, 322, 190-191.

Al-Khayat, H. A., Hudson, L., Reedy, M. K., Irving, T. C., \& Squire, J. M. (2003). Myosin head configuration in relaxed insect flight muscle: X-ray modeled resting cross-bridges in a pre-powerstroke state are poised for actin binding. Biophysical Journal, 85, 1063-1079.

Andrews, J., Meirer, F., Liu, Y., Mester, Z., \& Piero, P. (2011). Transmission X-ray microscopy for full-field nano-imaging of biomaterials. Microscopy Research and Technique, 74(7), 671-681. https://doi. org/10.1002/jemt.20907

Aristizábal-Torres, I. D. (2007). La resonancia magnética y sus aplicaciones en la agroindustria, una revisión. Revista Facultad Nacional de Agronomia-Medellin, 60(2), 4037-4066.

Betz, O., Wegst, U., Weide, D., Heethoff, M., Helfen, L., Lee, W. K., \& Cloetens, P. (2007). Imaging applications of synchrotron X-ray phase-contrast microtomography in biological morphology and biomaterials science. I. General aspects of the technique and its advantages in the analysis of millimetre-sized arthropod structure. Journal of Microscopy, 227(1), 51-71. https://doi.org/10.1111/j.13652818.2007.01785.x 
Bukejs, A., Bezděk, J., Alekseev, V. I., Kairišs, K., \& McKellar, R. C. (2020). Description of the male of fossil Calomicrus eocenicus Bukejs et Bezděk (Coleoptera: Chrysomelidae: Galerucinae) from Eocene Baltic amber using X-ray microtomography. Fossil Record, 19, 105-115. https://doi.org/10.5194/fr-23-105-2020

Büsse, S., \& Gorb, S. N. (2018). Material composition of the mouthpart cuticule in a damselfly larva (Insecta: Odonata) and its biomechanical significance. Royal Society Open Science, 5, 172117. http://doi.org/10.1098/ rsos. 172117

Büsse, S., Helmker, B., \& Hörnschemeyer, T. (2015). The thorax morphology of Epiophlebia (Insecta: Odonata) nymphs-including remarks on ontogenesis and evolution. Scientific Reports, 5, 12835. https://doi. org/10.1038/srep12835

Büsse, S., Hörnschemeyer, T., \& Fischer, C. (2016). Three-domensional reconstruction on cell level: Case study elucidates the ultrastructure of the spinning apparatus of Embia sp. (Insecta: Embioptera). Royal Society Open Science, 3, 160563. https://doi.org/10.1098/rsos.160563

Charlier, N., Desoil, M., Gossuin, Y., Gillis, P., \& Gallez, B. (2020). Electron paramagnetic resonance imaging of melanin in honey bee. Cell Biochemestry and Biophysycs. https://doi.org/10.1007/s12013-020-00903-8

Chitsaz, N., Marian, R., \& Chahl. J. (2020). Experimental method for 3D reconstruction of Odonata wings (methodology and dataset).PLOS ONE, 15(4), e0232193. https://doi.org/10.1371/journal.pone.0232193

Favila, M. E., Fresneau, D., Gonord, P., \& Ruaud, J. P. (2004). Nuclear magnetic resonance microscopy of the internal structure of the carrion rolling scarab canthon cyanellus cyanellus (Scarabaeidae: Scaracaeinae). The Coleopterists Bulletin, 58(1), 125-131. https://doi.org/10.1649/645

Ijiri, T., Todo, H., Hirabayashi, A., Kohiyama, K., \& Dobashi, Y. (2018). Digitization of natural objets with micro CT and photographs. PLOS ONE, 13(4), e0195852. https://doi.org/10.1371/journal.pone.0195852

Iwamoto, H. (2018). Synchrotron Radiation X-ray diffraction techniques applied to insect flight muscle. International Journal of Molecular Sciences, 19, 1784. https://doi.org/10.3390/ijms19061748

Keszthelyi, S., Pónya, Z., Csóka, A., Bázar, G., Morschhauser, T., \& Donkó, T. (2020a). Non-destructive imaging and spectroscopic techniques to investigate the hidden-lifestyle arthropod pests: A review. Journal of Plan Diseases and Protection, 127, 283-295. https://doi.org/10.1007/s41348-020-00300-6

Keszthelyi, S., Szöllösi, D., Strobel, L., Osváth, S., Szigeti, K., Pónya, Z., Csóka, A., \& Donkó, T. (2020b). Novel, X-ray supported kinetic imaging of hidden-lifestyle arthropods. Insect Science, 1-4. https://doi. org/10.1111/1744-7917.12753

Knupp, C., Morris, E., \& Squire, J. M. (2019). The interacting head motif structure does not explain the X-ray diffraction patterns in relaxed vertebrate (Bony fish) skeletal muscle and insect (Lethocerus) flight muscle. Biology, 8(67). https://doi.org/10.3390/biology8030067

Lee, W. K., \& Socha, J. J. (2009). Direct visualization of hemolymph flow in the heart of a grasshopper (Scistocerca americana). BMC Physiology, 9, 2. http://doi.org/10.1186/1472-6793-9-2

Magnotta, V. A., Smith, W. L. y Erkonen, W. E. (2014). Radiografía, tomografía computarizada, resonancia magnética y ecografía: principios e indicaciones, en W. L. Smith y T. A. Farrell (eds.), Introducción al diagnóstico por imagen (cuarta edición). Wolters Kluwer.

Márquez-Cardozo, C. J., Ramírez-Gallego, V. y Cartagena-Venezuela, J. R. (2009). Uso de rayos X para evaluar la maduración y deterioro de la pulpa en frutos de guanábaja (Annona muricata L. CV. Elita). Revista CES Medicina Veterinaria y Zootecnia, 4(2), 91-98.

Menezes, C., Vollet-Neto, A., Marsaioli, A. J., Zampieri, D., Fontoura, I. C., Luchessi, A. D., \& Imperatriz-Fonseca, V. L. (2015). A brazilian social bee must cultivate fungus to survive. Current Biology, 25, 2851-2855. http://dx.doi.org/10.1016/j.cub.2015.09.028 
Nelson, D. L. y Cox, M. M. (2009). Lehninger: principios de bioquimica (quinta edición). Barcelona: Ediciones OMEGA.

Rodríguez-Camacho, C., Prieto, B. y Bernal, J. (2011). Potenciales relacionados con eventos (PRE): aspectos generales conceptuales, en J. Silva-Pereyra (ed.). Métodos en neurociencias cognitivas. México: El Manual Moderno.

Rühr, R. T., \& Lambertz, M. (2019). Surface contrast enhancement of integumentary structures in X-ray tomography. Journal of Anatomy, 235, 379-385. https://doi.org/10.1111/joa.13008

Salinas, A. R., Arango-Perearmau, M. R., Galo, C. D. V., Alzugaray, C., Carnevale, N. J., Gibbons, R. y Craviotto, R. M. (2016). Manual de rayos X aplicado a la calidad de semillas. Argentina: Ediciones INTA.

Sarnat, E. M., Fischer, G., \& Economo, E. P. (2016). Inordinate Spinescence: Taxonomic Revision and Microtomography of the Pheidole cervicornis Species Group (Hymenoptera, Formicidae). PLOS ONE, 11(7), e0156709. https://doi.org/10.1371/journal.pone.0156709

Schilling, F., Dworschak, K., Schopf, R., Kühn, R., \& Glaser, S. J. (2012). Non-invasive lipid measurement in living insects using NMR microscopy. The Journal of Experimental Biology, 215, 3137-3141. https://doi. org/10.1242/jeb.071209

Seo, Y. (2018). High spatial resolution magnetic resonance imaging of insects covered with a hard exoskeleton. Concepts in Magnetic Resonance Part B, 48B(1), e21366. https://doi.org/10.1002/cmr.b.21366

Silva-Pereyra, J. (2011). Localización de fuentes de corriente de la actividad eléctrica cerebral: tomografía eléctrica cerebral, en J. Silva-Pereyra (ed.), Métodos en neurociencias cognitivas. México: El Manual moderno.

Triplehorn, C. A., \& Johnson, N. F. (2005). Borror and Delong's introduction to the study of insects (7th edition). USA: Thomson, Brooks/Cole.

Wakonig, K., Diaz, A., Bonnin, A., Stampanoni, M., Bergamaschi, A., Ihli, J., Guizar-Sicairos, M., \& Menzei, A. (2019). X-ray fourier ptychography. Science Advances, 5(2), https://doi.org/10.1126/sciadv.aav0282

Zhakypov, Z., Mori, K., Hosoda, K., \& Paik, J. (2019). Designing minimal and scalable insect-inspired multi-locomotion millirobots. Nature. https://doi.org/10.1038/s41586-019-1388-8

\section{BY-NC-ND}

